\title{
An Introduction to the Special Issue on Pollinators
}

\section{By Amy C. Ganguli and Jason P. Harmon}

$\mathrm{P}$ ollinators provide crucial ecosystem services to rangelands. However, pollinators are undergoing a number of challenges that threaten these services. Our intent in organizing and editing this special issue of Rangelands is to provide those working in rangeland ecosystems information about why pollination is important to rangelands, why rangelands are important to pollinators, and how these relationships are being threatened. Recently pollinators have garnered attention due to high-profile media coverage of honeybee population declines or collapses. This, coupled with increasing awareness of native pollinator decline, has paved the way for improved management resources for pollinator conservation efforts. Furthermore, federal conservation programs and other conservation-based incentive programs aimed at private lands will likely continue to increase their emphasis on maintaining, enhancing, and restoring pollinator habitat and pollinator populations in rangeland ecosystems.

This special issue on pollinators will describe the connections between pollinators and rangelands, including how rangelands enhance pollinators and the services pollinators provide as well as how pollinators benefit the objectives of rangeland managers.

We begin this discussion by presenting a brief overview of how pollination works in rangelands, focusing on how plants grow and reproduce in rangelands and why they bother with bribing animals to help them in this endeavor (Harmon et al.). Benefits of pollination extend well beyond plant reproduction and fitness. In the process of providing pollination services, pollinators are responsible for a host of additional ecosystem services. The importance of pollinators from a wildlife habitat perspective is discussed by Gilgert and Vaughan, along with examples of how rangeland management for pollinators can provide a useful framework for managing wildlife biodiversity. Pollinator diversity is also important in maintaining plant community diversity. Travers et al. highlight the importance of lepidopteran pollinators (moths and butterflies) and discuss how pollinators are important to the maintenance of rare plant species.

In efforts to bring the issues threatening rangeland pollinators and their habitat to light, Cane describes the challenges that pollinators face in the western United States with an emphasis on wild bees. Rangeland management for pollinators is more specifically discussed by Black et al., who provide general considerations to use when looking at common rangeland management practices such as grazing, prescribed burning, mowing, and herbicide use. Although each of these management practices can result in negative consequences for pollinators, they can also be applied in a manner that will benefit pollinators.

Pollinator services are also a large concern for specialty agricultural crops. In our final paper, Chaplin-Kramer et al. illustrate the value of rangeland for supplying pollination services to production agriculture in California.

Papers in this issue have been peer-reviewed. Because of space limitations, we limited the number of citations within each article. Interested readers can access supplemental literature and data authors have referenced at www.srmjournals. org. To read more about pollinators visit "Buzzing the Literature" (Solga) for recent literature.

\section{Acknowledgments}

We would like to thank the USDA-Natural Resource Conservation Service, The Xerces Society of Invertebrate Conservation, the USDA-Forest Service, the Great Basin Native Plant Selection and Increase Project, the US Department of Interior-Bureau of Land Management, and the Great Basin Restoration Initiative for sponsoring this special issue of Rangelands. In particular, we would like to express gratitude for the insight and contributions of Scott Black, Wendell Gilgert, Nancy Shaw, Mace Vaughn, and Lori Hidinger. We appreciate the reviewers who provided constructive comments that improved the quality of this issue, as well as the contributions of the invited authors.

Authors are Assistant Professor, Range Science Program, North Dakota State University, Fargo, ND 58108, USA (Ganguli); and Assistant Professor, Entomology Dept, North Dakota State University, Fargo, ND 58108, USA, Jason.Harmon@ndsu.edu (Harmon). 